\section{OPEN ACCESS}

Edited by:

Souhaila Al Khodor,

Sidra Medical and Research Center,

Qatar

Reviewed by:

Melanie Lisa Hutton,

Monash University, Australia

Paul Race,

University of Bristol, UK

*Correspondence:

Chih-Ho Lai

chlai@mail.cgu.edu.tw

${ }^{\dagger}$ These authors have contributed equally to this work

Received: 07 October 2016 Accepted: 19 December 2016 Published: 17 January 2017

Citation:

Liao W-C, Huang $M-Z$, Wang $M L$ Lin C-J, Lu T-L, Lo H-R, Pan Y-J, Sun Y-C, Kao M-C, Lim H-J and

Lai C-H (2017) Statin Decreases Helicobacter pylori Burden in Macrophages by Promoting Autophagy.

Front. Cell. Infect. Microbiol. 6:203. doi: 10.3389/fcimb.2016.00203

\title{
Statin Decreases Helicobacter pylori Burden in Macrophages by Promoting Autophagy
}

\begin{abstract}
Wei-Chih Liao ${ }^{1,2 \dagger}$, Mei-Zi Huang $3,4+$, Michelle Lily Wang ${ }^{5}$, Chun-Jung Lin ${ }^{5,6}$, Tzu-Li Lu ${ }^{3}$, Horng-Ren Lo ${ }^{7}$, Yi-Jiun Pan ${ }^{5}$, Yu-Chen Sun ${ }^{8}$, Min-Chuan Kao ${ }^{4}$, Hui-Jing Lim ${ }^{4}$ and Chih-Ho Lai ${ }^{4,5,9,10 *}$

${ }^{1}$ Graduate Institute of Clinical Medical Science, China Medical University, Taichung, Taiwan, ${ }^{2}$ Department of Pulmonary and Critical Care Medicine, China Medical University Hospital, Taichung, Taiwan, ${ }^{3}$ Department of Medical Laboratory Science and Biotechnology, China Medical University, Taichung, Taiwan, ${ }^{4}$ Department of Microbiology and Immunology, Graduate Institute of Biomedical Sciences, College of Medicine, Chang Gung University, Taoyuan, Taiwan, ${ }^{5}$ Graduate Institute of Basic Medical Science, School of Medicine, China Medical University, Taichung, Taiwan, ${ }^{6}$ Department of Urology, University of Texas Southwestern Medical Center, Dallas, TX, USA, ${ }^{7}$ Department of Medical Laboratory Science and Biotechnology, Fooyin University, Kaohsiung, Taiwan, ${ }^{8}$ Department of Laboratory Medicine, Chang Gung Memorial Hospital, Taoyuan, Taiwan, ${ }^{9}$ Department of Nursing, Asia University, Taichung, Taiwan, ${ }^{10}$ Department of Pediatrics, Molecular Infectious Disease Research Center, Chang Gung Children's Hospital and Chang Gung Memorial Hospital, Taoyuan, Taiwan
\end{abstract}

Statins, 3-hydroxy-3-methyl-glutaryl-coenzyme A (HMG-CoA) reductase inhibitors, have been found to provide protective effects against several bacterial infectious diseases. Although the use of statins has been shown to enhance antimicrobial treated Helicobacter pylori eradication and reduce $\mathrm{H}$. pylori-mediated inflammation, the mechanisms underlying these effects remain unclear. In this study, in vitro and ex vivo macrophage models were established to investigate the molecular pathways involved in statin-mediated inhibition of $H$. pylori-induced inflammation. Our study showed that statin treatment resulted in a dose-dependent decrease in intracellular $H$. pylori burden in both RAW264.7 macrophage cells and murine peritoneal exudate macrophages (PEMs). Furthermore, statin yielded enhanced early endosome maturation and subsequent activation of the autophagy pathway, which promotes lysosomal fusion resulting in degradation of sequestered bacteria, and in turn attenuates interleukin (IL)- $1 \beta$ production. These results indicate that statin not only reduces cellular cholesterol but also decreases the $H$. pylori burden in macrophages by promoting autophagy, consequently alleviating H. pylori-induced inflammation.

Keywords: autophagy, cholesterol, Helicobacter pylori, HMG-CoA reductase, statin

\section{INTRODUCTION}

Helicobacter pylori is a Gram-negative microaerophilic spirochete that colonizes the human stomach and is estimated to have infected greater than half of the global population (Marshall, 2002). Persistent $H$. pylori infection is associated with several upper gastrointestinal disorders such as gastritis, peptic ulcers, and gastric adenocarcinoma (Wroblewski et al., 2010).

Although $\mathrm{H}$. pylori is generally considered an intracellular pathogen, this organism lives in the mucosal layer and tightly adheres to the gastric epithelial surface. Notably, virulent strains of $H$. pylori, can delay uptake and promote the formation of megasomes within macrophages, 
which comprises a crucial feature of $H$. pylori-induced pathogenesis (Allen et al., 2000). Moreover, cholesterol$\alpha$-glucosyltransferase, which is responsible for cholesterol glucosylation in macrophages, was found to contribute to the protection of $H$. pylori from phagocytosis (Wunder et al., 2006). These lines of evidence suggest that $H$. pylori can survive intracellularly within specific compartments of macrophages to avoid phagocytosis-mediated killing.

The inhibitors of 3-hydroxy-3-methyl-glutaryl-coenzyme A (HMG-CoA) reductase, commonly known as statins, are widely prescribed for lowering serum cholesterol (Armitage, 2007). Notably, statins have also been shown to reduce the risk of severe bacterial infections, including infections by Chlamydia pneumoniae (Erkkilä et al., 2005), Clostridium difficile (MotzkusFeagans et al., 2012), Staphylococcus aureus (Chow et al., 2010), and Streptococcus pneumoniae (Boyd et al., 2012). However, the immunomodulatory properties of statins provide only a partial explanation for the mechanism by which these compounds inhibit bacterial infections (Jain and Ridker, 2005).

The human immune system employs various mechanisms to inhibit bacterial infections. While autophagy is a cell process that typically functions as a recycling pathway, degrading nonfunctional and unnecessary components and rearranging these components to support cellular survival (Mariño et al., 2014), this process was also found to contribute to immune defense by degrading invading pathogens (Mizushima et al., 2008; Zhao et al., 2008). As such, these findings indicate that stimulation of cellular autophagy may attenuate $H$. pyloriinduced pathogenesis (Yang and Chien, 2009).

Antimicrobial agents, particularly a triple therapy regimen consisting of a proton-pump inhibitor, amoxicillin, and clarithromycin, are the most effective means of eradicating $H$. pylori infections (O'Connor et al., 2013). Although the cure rate varies between countries, the triple therapy regimen remains the recommended treatment for $H$. pylori infection ( $O^{\prime}$ Connor et al., 2013). Notably, the administration of this triple therapy regimen along with statins has been shown to accelerate the clearance of $H$. pylori and reduce $H$. pylori-related inflammation (Tariq et al., 2007; Yamato et al., 2007; Nseir et al., 2012). However, the molecular mechanisms underlying the regulatory effects of statins on $H$. pylori-induced pathogenesis require further investigation. In this study, we first hypothesized that statins may influence the immune response via upregulation of autophagy and attenuation of $H$. pylori-induced inflammation. We utilized in vitro and ex vivo macrophage models of $H$. pylori infection to investigate the mechanism underlying the statin-mediated mitigation of $H$. pylori pathogenesis. We also explored how statin influences the bacterial burden and reduces inflammation by upregulating cellular autophagy and consequently alleviating H. pylori-associated pathogenesis.

\section{MATERIALS AND METHODS}

\section{Antibodies and Reagents}

The light chain 3 (LC3)-specific monoclonal antibody was purchased from Cell Signaling Technology (Danvers, MA), while the rabbit antibodies against SQSTM1/p62 and beclin-1 were purchased from GeneTex (Irvine, CA). The rabbit antiearly endosome antigen-1 (EEA-1), anti-lysosome-associated membrane protein-1 (LAMP-1), and mouse monoclonal anti$\beta$-actin antibodies were purchased from Abcam (Cambridge, UK), Abgent (San Diego, CA), and Santa Cruz Biotechnology (Dallas, TX), respectively. Simvastatin was purchased from Sigma-Aldrich (St. Louis, MO).

\section{Bacterial and Cell Culture}

H. pylori 26695 (ATCC 700392) was cultured on 10\% sheep blood agar plates in a microaerophilic environment $\left(10 \% \mathrm{CO}_{2}\right.$, $5 \% \mathrm{O}_{2}$, and $85 \% \mathrm{~N}_{2}$ ) at $37^{\circ} \mathrm{C}$. Cultures were incubated for $24-$ $36 \mathrm{~h}$ to achieve optimum microbial activity (Lai et al., 2005). Murine RAW264.7 macrophages (ATCC TIB-71) were cultured in Dulbecco's Modified Eagle Medium (DMEM) supplemented with $10 \%$ endotoxin-free fetal bovine serum (HyClone, Logan, UT).

\section{Analysis of Cellular Cholesterol and Cytotoxicity}

RAW264.7 cells were treated with simvastatin $(0,5$, or $10 \mu \mathrm{M})$ at $37^{\circ} \mathrm{C}$ for $24 \mathrm{~h}$. Untreated cells were utilized as a control. The cellular cholesterol content of each treatment group was then evaluated using an Amplex Red cholesterol assay kit (Molecular Probes, Carlsbad, CA). The percentage of cellular cholesterol was determined as follows: (fluorescence of treated cells obtained from a standard curve/total fluorescence of untreated cells) $\times 100 \%$. Meanwhile, the viability of RAW264.7 cells was determined via the trypan blue exclusion assay, as described previously (Lai et al., 2008).

\section{Preparation of Murine Peritoneal Exudate Macrophages (PEMs)}

PEMs isolated from C57BL/6 mice were used to investigate the effects of simvastatin on $H$. pylori-induced autophagy. Mice were maintained at the Animal Center of Chang Gung University (Taoyuan, Taiwan). All procedures were performed according to the "Guide for the Care and Use of Laboratory Animals" (NRC, USA) and were approved by the Animal Experimental Committee of Chang Gung University. PEMs were prepared after euthanasia by lavaging mice with an intraperitoneal injection of 3\% thioglycolate, as described previously (Lu et al., 2012a). Harvested cells were then seeded into 24 -well tissue culture plates and incubated at $37^{\circ} \mathrm{C}$ for $2 \mathrm{~h}$, after which non-adherent cells were removed. Adherent cells were then treated with simvastatin and/or $H$. pylori and subjected to bacterial intracellular survival assay and western blot analyses.

\section{Phagocytosis Assay}

RAW264.7 cells were treated with PBS or simvastatin (0, 5, or $10 \mu \mathrm{M}$ ) for $8 \mathrm{~h}$ and incubated with latex beads (IgG-FITC complex) at a ratio of 1:100, according to the manufacturer's instructions (Cayman, Ann Arbor, MI). After incubation for $1 \mathrm{~h}$, the treated cells were washed with PBS, fixed in $3.7 \%$ paraformaldehyde, and then subjected to flow cytometry analysis. 


\section{Bacterial Adhesion Assay}

The numbers of cell-associated bacteria were measured as described previously (Lai et al., 2008). Briefly, RAW264.7 cells were treated with PBS or $10 \mu \mathrm{M}$ simvastatin for $8 \mathrm{~h}$ and then infected with $H$. pylori at a multiplicity of infection (MOI) of 100 for $6 \mathrm{~h}$. Infected cells were washed three times to remove unbound bacteria and then lysed with distilled water for $10 \mathrm{~min}$. Lysates were diluted in PBS, then plated onto Brucella blood agar plates and cultured for 3-5 days. Viable bacteria were counted and expressed as colony-forming units (CFU).

\section{Bacterial Intracellular Survival Assay}

RAW264.7 cells and murine PEMs and cells were not treated or treated with $10 \mu \mathrm{M}$ simvastatin for $8 \mathrm{~h}$ and then infected with $H$. pylori $(\mathrm{MOI}=100)$ for $16 \mathrm{~h}$. Cells were treated with gentamycin $(100 \mu \mathrm{g} / \mathrm{ml})$ to eradicate the extracellular bacteria (Lai et al., 2006). The H. pylori-infected cells were washed with PBS three times and then incubated in sterile water at $37^{\circ} \mathrm{C}$ for $10 \mathrm{~min}$ to osmotically disrupt the cell membrane. The resulting lysates were then diluted in PBS and applied to Brucella blood agar plates. Viable $H$. pylori colonies were enumerated after 3-5 days of incubation and were presented as CFU.

\section{Western Blot Analysis}

To analyze protein expression levels, RAW264.7 cells or PEMs were treated with simvastatin $(0,5$, or $10 \mu \mathrm{M})$ at $37^{\circ} \mathrm{C}$ for $8 \mathrm{~h}$ prior to infection with $H$. pylori for $16 \mathrm{~h}$. Cell lysates were prepared and boiled with sample dye at $100^{\circ} \mathrm{C}$ for $5 \mathrm{~min}$. Samples were separated by $10-12 \%$ sodium dodecyl sulfatepolyacrylamide gel electrophoresis (SDS-PAGE) and transferred to polyvinylidene fluoride membranes (Millipore, Billerica, MA). Membranes were blocked by incubation in TBS-T (Tris-buffered saline containing $0.1 \%$ Tween 20 ) containing 5\% skim milk at room temperature for $1.5 \mathrm{~h}$, and then probed with primary antibodies specific to autophagy-related proteins or $\beta$-actin $(0.2 \mu \mathrm{g} / \mathrm{ml})$, respectively, at $4^{\circ} \mathrm{C}$ overnight. Probed membranes were washed with TBS-T and incubated with horseradish peroxidase-conjugated secondary antibodies $(0.1 \mu \mathrm{g} / \mathrm{ml})$ (Santa Cruz Biotechnology) at room temperature for $1 \mathrm{~h}$, and proteins of interests were visualized using $\mathrm{ECL}^{\mathrm{TM}}$ western blotting Detection Reagents (GE Healthcare, Little Chalfont, UK), and an ImageQuant LAS-4000 system (GE Healthcare). Protein expression levels were quantified using UN-SCAN-IT gel 6.1 software (Silk Scientific Corporation, Orem, UT).

\section{Immunofluorescence Microscopy}

To visualize the co-localization of autophagosome fused with lysosome, cells were subjected to immunofluorescence microscopy analysis (Parihar et al., 2014). RAW264.7 cells (2 $\times 10^{6}$ ) were seeded on cover glass in 6-well plates, treated with $10 \mu \mathrm{M}$ simvastatin, and co-incubated with $H$. pylori (MOI $=100$ ) for $16 \mathrm{~h}$. To observe the early to late stages of autophagosome formation, the cells were probed using a Cyto$\mathrm{ID}^{\mathrm{TM}}$ autophagy detection kit (Enzo Life Sciences, Villeurbanne, France; Chan et al., 2012). Meanwhile, the formation of lysosome was observed using a Cell Navigator ${ }^{\mathrm{TM}}$ Lysosome Staining Kit (AAT Bioquest, Sunnyvale, CA). Fluorescence signals from $H$. pylori-infected macrophages were visualized by confocal laserscanning microscopy (Zeiss LSM 780; Carl Zeiss, Oberkochen, Germany).

\section{Enzyme-Linked Immunosorbent Assay (ELISA)}

After treating with $10 \mu \mathrm{M}$ simvastatin and infecting with H. pylori, RAW264.7 cell supernatants were collected and interleukin (IL)-1 $\beta$ secretion was analyzed using a mouse IL$1 \beta$ ELISA kit, according to the manufacturer's instructions (Invitrogen, Waltham, MA).

\section{Statistical Analysis}

Experimental results are expressed as means \pm standard errors of the mean (SEM). Differences in results between groups were evaluated using Student's $t$-tests. For analyses of variance, oneway analysis of variance (ANOVA) was utilized. $P<0.01$ were considered statistically significant. Statistical analyses were performed using SPSS version 11.0 software (SPSS Statistics, Inc., Chicago, IL).

\section{RESULTS}

\section{Statin Reduces the Intracellular Burden of $H$. pylori in Macrophages}

Statins are known as inhibitors of HMG-CoA reductase, which is the rate-limiting enzyme of the mevalonate pathway (Armitage, 2007). In this study, we first evaluated whether simvastatin is capable of reducing the cellular cholesterol of macrophages. As shown in Figure 1A, there was a dose-dependent reduction in the level of cellular cholesterol in RAW264.7 macrophage cells treated with simvastatin (Figure 1A). We then assessed whether simvastatin treatment affected macrophage or bacterial viability. Notably, both macrophages and $H$. pylori remained viable, even after treatment with $10 \mu \mathrm{M}$ simvastatin (Figure 1B). These results indicate that simvastatin reduces the levels of cellular cholesterol in macrophages without affecting cell viability or bacterial survival.

To further validate whether statin-treated macrophages function similarly to untreated macrophages, RAW264.7 cells were untreated or treated with statin $(10 \mu \mathrm{M})$ and subjecting to the latex bead phagocytosis assay. As shown in Figure S1, macrophages treated with statin exhibited similar levels of phagocytosis to the untreated cells. Likewise, statin treatment had no significant effect on extracellular bacterial adhesion (Figure S2). Together, these results demonstrate that macrophages retain their normal functions in the presence of statins.

To investigate whether simvastatin inhibits $H$. pylori survival in macrophages, RAW264.7 cells were pretreated with simvastatin for $8 \mathrm{~h}$ and infected with $H$. pylori $(\mathrm{MOI}=100)$ for an additional $16 \mathrm{~h}$. As shown in Figure 1C, treatment with 5 and $10 \mu \mathrm{M}$ simvastatin resulted in significant dose-dependent reductions in $H$. pylori burden in RAW264.7 cells. To further explore the inhibitory effects of simvastatin on intracellular bacterial survival, we performed an ex vivo analysis using murine PEMs isolated from C57BL/6 mice. As observed in RAW264.7 

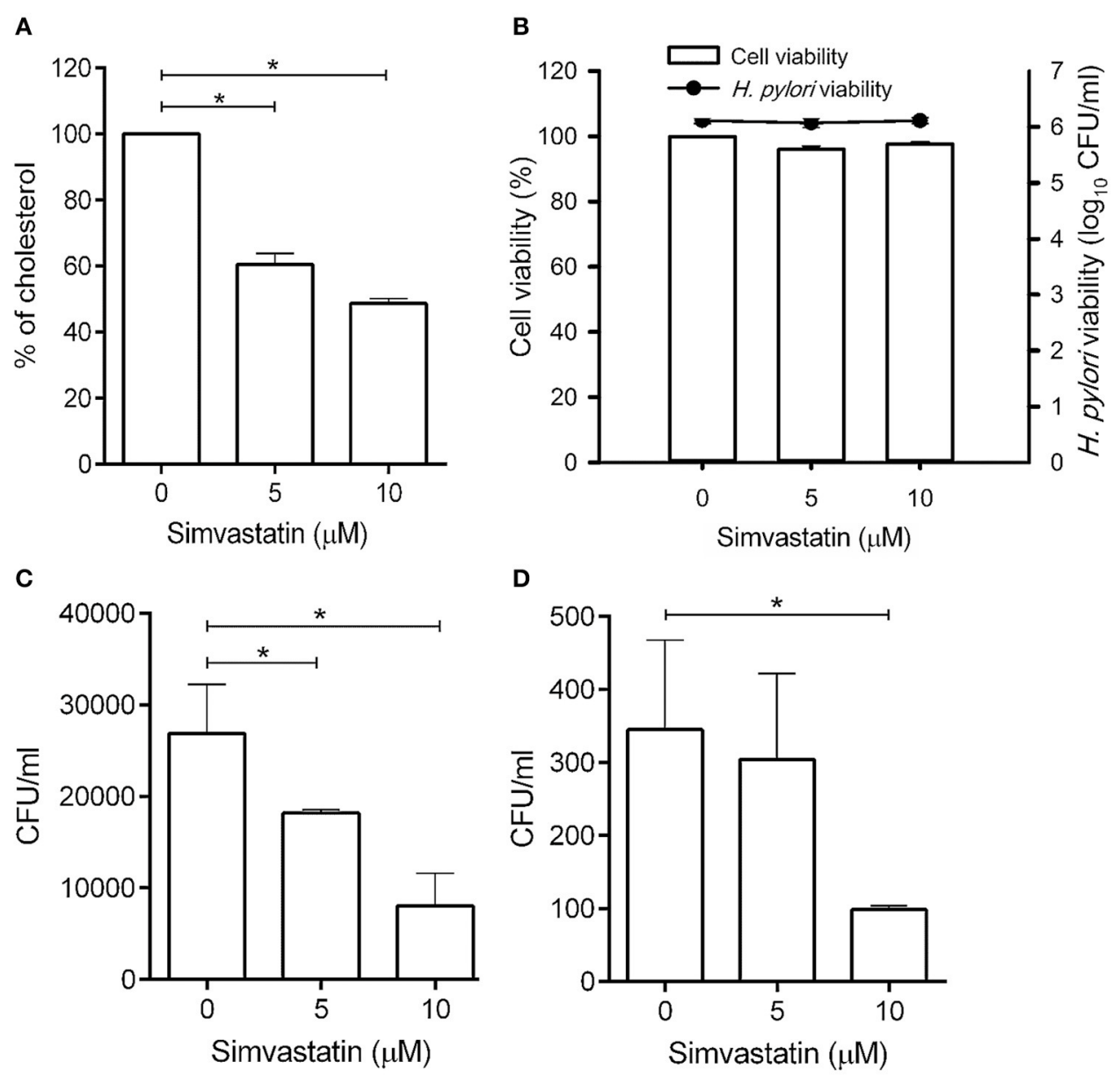

FIGURE 1 | Statin treatment reduces cellular cholesterol and decreases the intracellular bacterial burden in macrophages. (A) RAW264.7 cells were treated with various concentrations of simvastatin $(0,5$, or $10 \mu \mathrm{M})$ for $24 \mathrm{~h}$ and subjected to cellular cholesterol assay analysis. (B) RAW264.7 and Helicobacter pylori cultures were treated with 0,5 , or $10 \mu \mathrm{M}$ simvastatin for $24 \mathrm{~h}$. Macrophage cell viability was then assessed by trypan blue exclusion assay analysis and bacterial viability was determined by counting the number of colony-forming units (CFU) for each treatment group on blood agar plates. (C) RAW264.7 cells and (D) murine peritoneal exudate macrophages (PEMs) were pretreated with simvastatin $(0,5$, or $10 \mu \mathrm{M})$ for $8 \mathrm{~h}$ and infected with $\mathrm{H}$. pylori at a MOI of 100 for $16 \mathrm{~h}$. The numbers of viable intracellular bacteria were then determined via gentamicin protection assays and were expressed as the number of viable CFU. Statistical significance was evaluated using Student's $t$-test. ${ }^{*} P<0.01$.

cells, PEMs that were pretreated with 5 or $10 \mu \mathrm{M}$ simvastatin for $8 \mathrm{~h}$ exhibited significant reductions in $H$. pylori burden after $16 \mathrm{~h}$ of infection compared to the control population (Figure 1D). These results demonstrate that simvastatin decreases the intracellular $H$. pylori burden in both in RAW264.7 and primary murine PEMs in a dose-dependent manner.

\section{Statin Promotes H. Pylori-Induced Autophagy in Macrophages}

Because treatment of cells with cholesterol-lowering agents has been shown to induce autophagy (Cheng et al., 2006; de Chastellier and Thilo, 2006), we investigated whether statin influences the immune response by upregulating autophagy and attenuating $H$. pylori-induced inflammation. To address this question, we established a macrophage infection model to explore the mechanisms involved in the inhibition of $H$. pylori-induced inflammation by statin. Following the induction of autophagy, the microtubule-associated protein LC3 is converted from LC3 I to LC3-II. As such, the expression of LC3-II is considered a marker of autophagy (Klionsky et al., 2012). We therefore evaluated the expression levels of this protein, as well as those of EEA-1, an early marker localized on phagosomal membranes, and LAMP-1, a marker localized on lysosomal membranes (Fratti et al., 2001; Huynh et al., 2007; Parihar et al., 2014), in RAW264.7 cells infected with $H$. pylori for $0-48$ h by western blot analysis. As shown in Figure $\mathbf{2 A}$, there was a gradual increase in the levels of $H$. pylori-induced EEA-1 and LAMP-1 expression, and a concurrent increase in the conversion of LC3-I to LC3-II, over the course of the infection. Peak levels of conversion from LC3-I to LC3-II were observed between 16 and $36 \mathrm{~h}$. Next, we evaluated the expression of the autophagy-related proteins beclin-1 and SQSTM1/p62, which are known to participate in the initiation of autophagy with LC3-II (Kang et al., 2011; Levine et al., 2011). RAW264.7 cells treated with simvastatin $(0,5$, or $10 \mu \mathrm{M})$ and 
infected with $H$. pylori for $16 \mathrm{~h}$ exhibited slightly increased expression of beclin-1 and p62 (Figure 2B). To confirm these findings, we subsequently analyzed the effects of simvastatin on PEMs isolated from C57BL/6 mice. As shown in Figure 2C, we observed increased levels in the conversion of LC3-I to LC3-II, as well as increased expression of beclin-1 and p62, in H. pyloriinfected PEMs treated with simvastatin, compared to untreated cells. These results suggest that simvastatin treatment not only reduces cellular cholesterol but also promotes $H$. pylori-induced autophagy in macrophages.

\section{Statin Facilitates Autophagosome Formation in $\mathbf{H}$. pylori-Infected Macrophages}

To further evaluate the effects of simvastatin on macrophages, RAW264.7 cells were stained with Cyto-ID autophagy green dye for visualization of autophagosomes and examined by confocal microscopy. The control group (untreated and uninfected) exhibited faint Cyto-ID green fluorescence (punctaformation; Figure 3A), while cells treated with simvastatin or H. pylori alone showed only moderate staining. In contrast, cells that were treated with simvastatin and subsequently infected with $H$. pylori exhibited significantly increased autophagosome formation compared to both the control group and to the cells treated with simvastatin or $H$. pylori alone (Figure 3B). These results demonstrate that simvastatin enhances $H$. pylori-induced autophagosome formation in macrophages.

\section{Statin Promotes Autophagosome and Lysosome Fusion in $\mathbf{H}$. pylori-Infected Macrophages}

Mature autophagosomes fuse with lysosomes to form autolysosomes, which contain lysosomal enzymes capable of degrading sequestered bacteria (Deen et al., 2013). We thus investigated whether statin treatment promotes bacterial degradation in macrophages by inducing autophagosome maturation and fusion with lysosomes. As shown in Figure 4A, RAW264.7 cells treated with either simvastatin or $H$. pylori alone exhibited little co-localization of Cyto-ID and lysosomal fluorescence within the cytoplasm. Conversely, cells treated with simvastatin and then infected with $H$. pylori exhibited marked co-localization of Cyto-ID and lysosomes (Figure 4B). We also tested whether statin treatment promoted $H$. pylori-induced lysosome formation. As shown in Figure 5A, treatment with both simvastatin and $H$. pylori resulted in increases in EEA-1 and LAMP-1 expression, compared with cells exposed to simvastatin or $H$. pylori alone. We then sought to analyze whether statin reduces the bacterial burden, and whether this had any effect on IL-1 $\beta$ production. The induction of IL- $1 \beta$ was dramatically increased in cells infected with $H$. pylori alone (Figure 5B). In contrast, when cells were treated with $10 \mu \mathrm{M}$ simvastatin and then infected with $H$. pylori, there was a significant decrease in the IL-1 $\beta$ secretion compared with the cells exposed to $H$. pylori. Collectively, our results reveal that statin treatment facilitated $H$. pylori-induced autophagy, and subsequently promoted
A

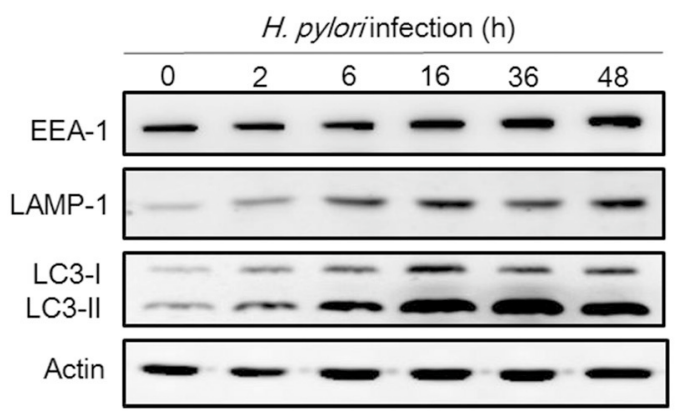

B

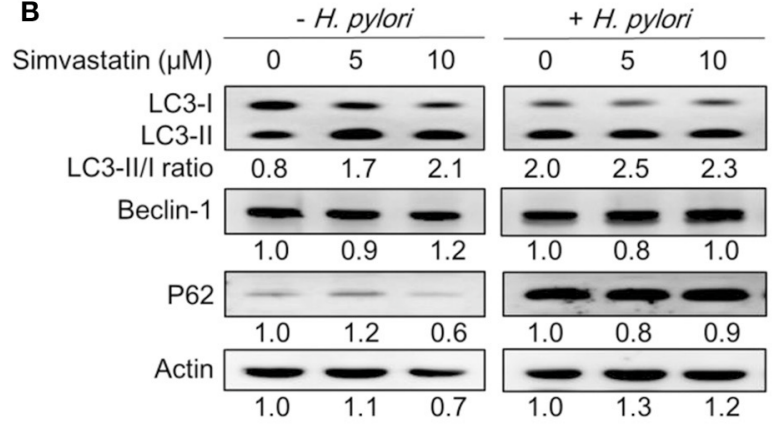

C
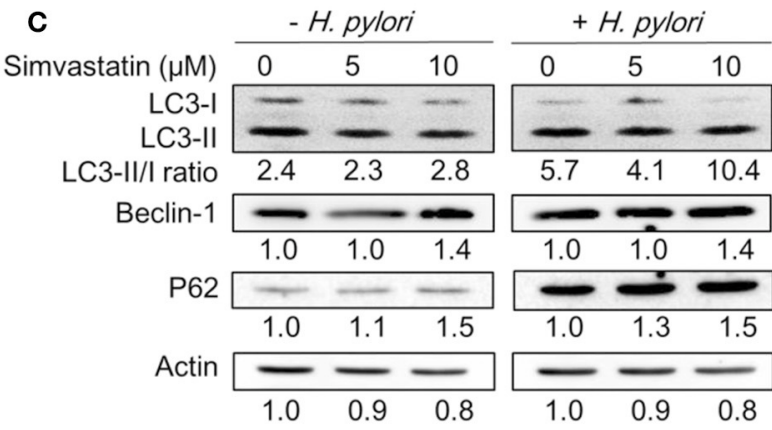

FIGURE 2 | Statin increases Helicobacter pylori-induced autophagy in macrophages. (A) RAW264.7 cells were infected with $\mathrm{H}$. pylori at a MOI of 100 for the indicated times $(0,2,616,36$, and $48 \mathrm{~h})$. The expression levels of proteins were then evaluated by western blot analysis. (B) RAW264.7 cells and (C) peritoneal exudate macrophages (PEMs) were pretreated with simvastatin $(0,5$, or $10 \mu \mathrm{M})$ for $8 \mathrm{~h}$ and then infected with $\mathrm{H}$. pylori at a $\mathrm{MOI}$ of 100 for an additional $16 \mathrm{~h}$. Cell lysates were then prepared and subjected to western blot analysis for detection of autophagy-associated proteins. $\beta$-actin expression levels were used as the protein loading control. Representative western blot results from one of two independent experiments are shown. The expression level of each protein was quantified by analysis of signal intensity, and normalized with that of $\beta$-actin. The expression level of each respective protein, relative to that of the control group, is indicated at the bottom of each lane.

autophagosome maturation and fusion with lysosomes, thereby reducing the bacterial burden in macrophages and ameliorating the inflammatory response.

\section{DISCUSSION}

Cholesterol-enriched microdomains, which provide platforms for microbial infection, are thought to be associated with 
A

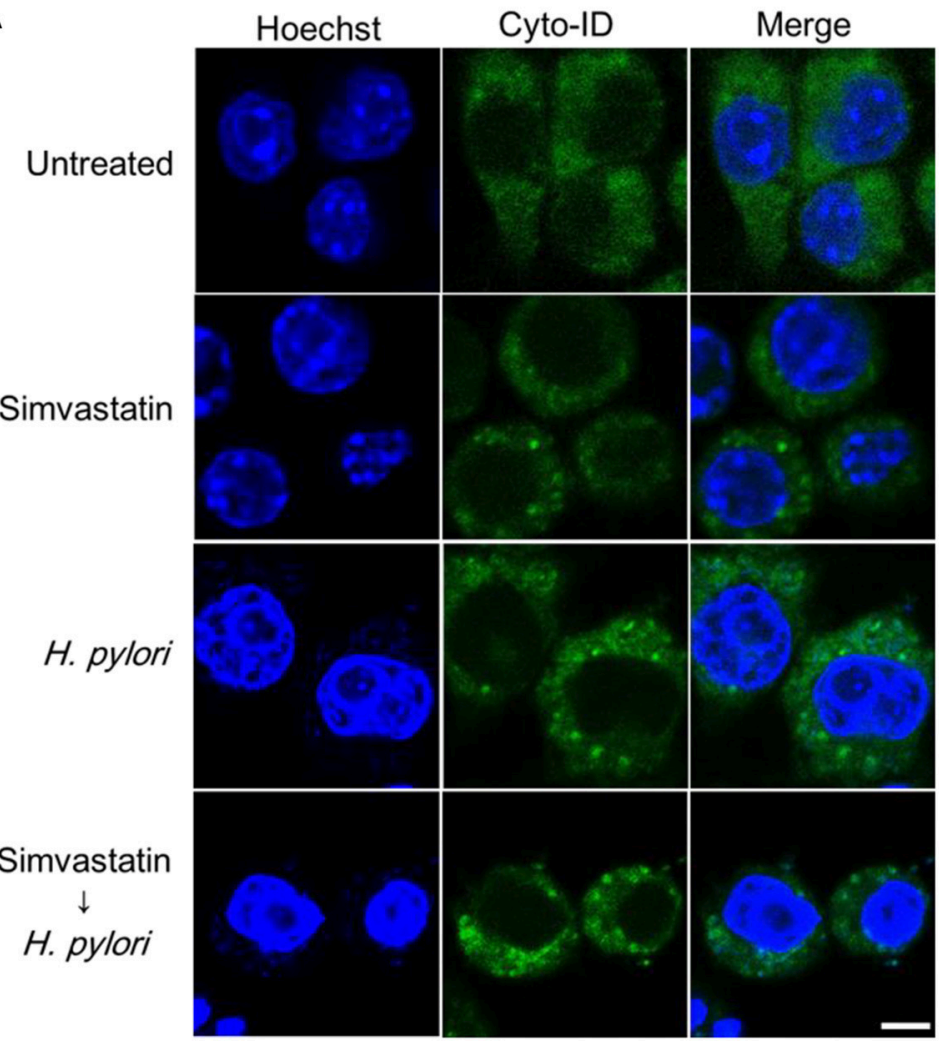

B

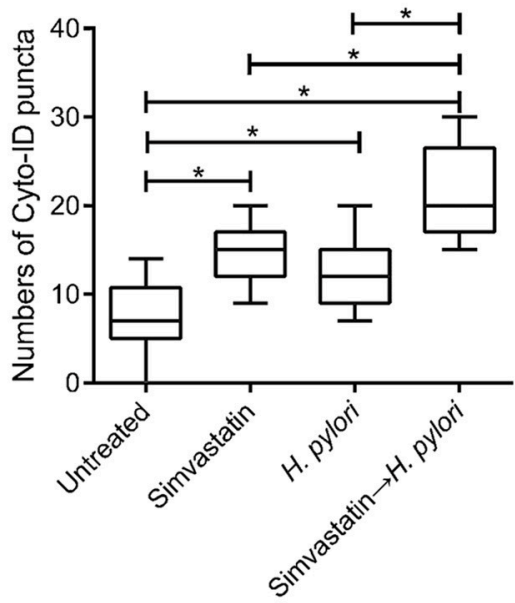

FIGURE 3 | Statin enhances autophagosome formation in Helicobacter pylori-infected macrophages. (A) Untreated RAW264.7 cells and cells treated with $10 \mu \mathrm{M}$ simvastatin were infected with or without $\mathrm{H}$. pylori at a $\mathrm{MOI}$ of 100 . After incubation for $16 \mathrm{~h}$, the cells were fixed and stained with Cyto-ID and Hoechst 33342 for detection of autophagosomes (green) and visualization of nuclei (blue), respectively. Stained cells were analyzed by confocal microscopy. Scale bar, $5 \mu \mathrm{m}$. (B) Box plots summarizing the number of Cyto-ID puncta in each cell (50 cells were evaluated per sample). Statistical significance was evaluated using Student's $t$-test. ${ }^{\star} P<$ 0.01 .

various infectious bacterial and viral diseases (Lin et al., 2015). In particular, cholesterol plays a crucial role in $H$. pylori cell invasion and virulence (Lai et al., 2013). Depletion of cellular cholesterol not only attenuates $H$. pylori-induced pathogenesis (Hutton et al., 2010; Lai et al., 2011) but also promotes autophagy (Cheng et al., 2006), which has been shown to contribute to immune defense against invading pathogens (Levine et al., 2011; Deretic et al., 2013; Lai et al., 2015). Statins, inhibitors of HMG-CoA reductase that are widely prescribed for lowering serum cholesterol, have also been employed to reduce the risk of certain bacterial infections (Chow et al., 2010; Nseir et al., 2010; Boyd et al., 2012; Motzkus-Feagans et al., 2012). The in vitro and ex vivo approaches utilized in this study provided evidence that statin treatment results in reduced bacterial burden in macrophages, and consequently attenuation of $H$. pylori pathogenesis, via activation of autophagy. These findings suggest that such cholesterol modulation could comprise a novel therapeutic approach for controlling $H$. pylori-associated diseases.

Previous studies have shown that $H$. pylori actively delays its uptake by macrophages, inhibits phagosome maturation via a VacA and urease-dependent mechanism, and resides in large vacuoles called megasomes (Allen et al., 2000; Schwartz and Allen, 2006). Another unusual feature of $H$. pylori is a requirement for cholesterol, which is acquired from host cell membranes and incorporated into the bacterium as cholesteryl glucosides (Wunder et al., 2006). In addition, it has been reported that $H$. pylori encodes several virulence factors that exploit cholesterol to gain a foothold in the host niche (Murata-Kamiya et al., 2010; Lai et al., 2013). Meanwhile, surface molecules located within the cholesterol-rich microdomains of host cells sense and respond to $H$. pylori in an orchestrated manner (Lu et al., 2012b; Lin et al., 2016). As such, both host- and pathogen-derived factors play critical roles in disease progression. In previous studies, we demonstrated that reduced cellular cholesterol resulted in reduced VacA activity, as well as attenuated CagA-induced inflammation and decreased bacterial survival, in $H$. pyloriinfected gastric epithelial cells (Lai et al., 2008, 2011; Wang et al., 2012). Specifically, reductions in cholesterol disrupt the integrity of lipid rafts, resulting in decreased type IV secretion system-mediated translocation of CagA into host cells (Hutton et al., 2010), thereby reducing downstream signaling and attenuating the inflammatory response (Lai 


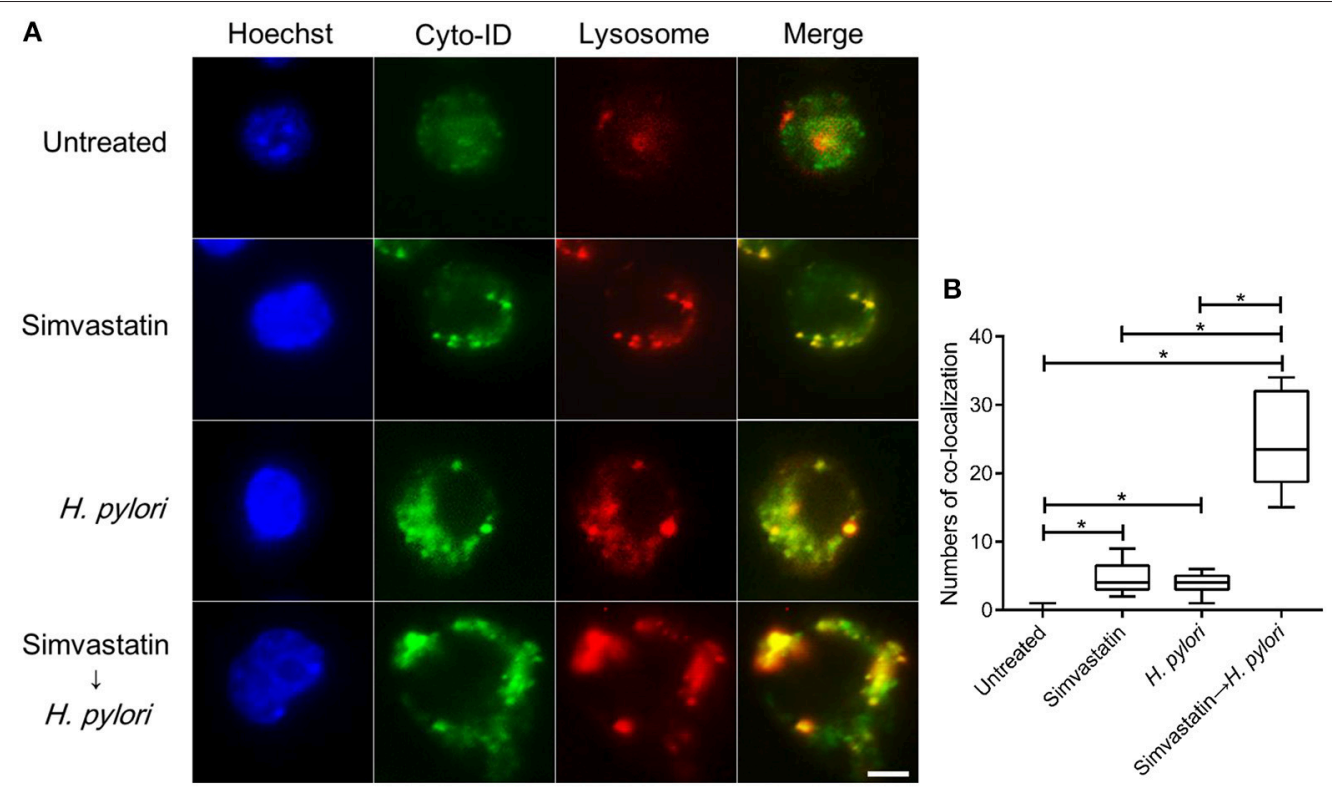

FIGURE 4 | Statin facilitates the fusion of autophagosomes and lysosomes in Helicobacter pylori-infected macrophages. (A) Untreated RAW264.7 cells and cells treated with $10 \mu \mathrm{M}$ simvastatin were infected with or without $\mathrm{H}$. pylori at a MOI of 100 for $16 \mathrm{~h}$. Cells were then fixed, stained with Cyto-ID (green), Lysosome Staining (red), and Hoechst 33342 (blue) for visualization of autophagosomes, lysosomes, and nuclei, respectively, and analyzed by confocal microscopy. Scale bar, $5 \mu \mathrm{m}$. (B) Box plots summarizing the numbers of co-localized Cyto-ID puncta and lysosomes in each cell (50 cells were evaluated per sample). Statistical significance was evaluated using Student's $t$-test. ${ }^{*} P<0.01$.
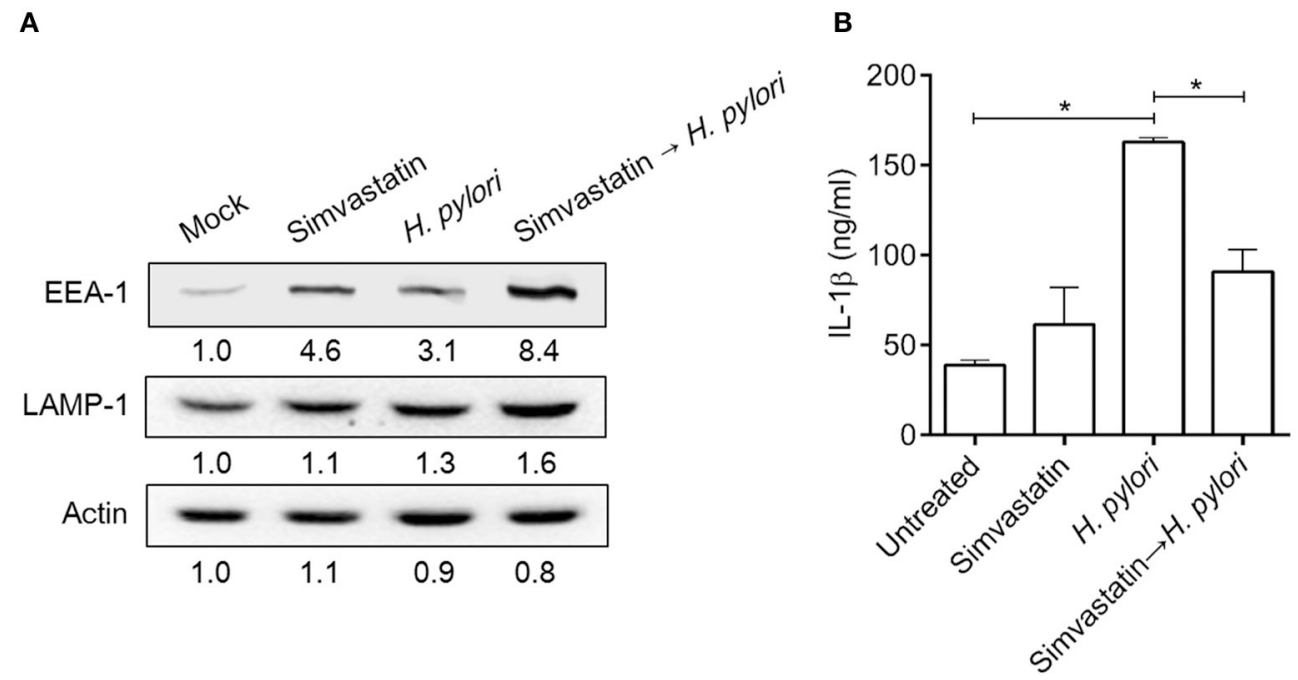

FIGURE 5 | Statin induces lysosome formation and mitigates proinflammatory cytokine production in Helicobacter pylori-infected macrophages. RAW264.7 cells were treated with $10 \mu \mathrm{M}$ simvastatin for $8 \mathrm{~h}$ and then infected with $\mathrm{H}$. pylori at a MOI of 100 for an additional $16 \mathrm{~h}$. (A) The expression levels of EEA-1 and LAMP-1 were analyzed by western blot. Representative western blot results from one of two independent experiments are shown. The expression level of each protein was quantified by analysis of signal intensity, and normalized to that of $\beta$-actin. The expression level of each respective protein, relative to that of the control group, is indicated at the bottom of each lane. (B) The levels of IL-1 $\beta$ present in each culture supernatant were determined by enzyme-linked immunosorbent assay (ELISA). Statistical significance was evaluated using Student's $t$-test. ${ }^{\star} P<0.01$.

et al., 2011). In the current study, we demonstrate that statin treatment yielded enhanced autophagy and reduced bacterial burdens in macrophages, followed by decreased levels of $H$. pylori-induced IL-1 $\beta$ production, suggesting that statin may attenuate $H$. pylori-induced pathogenesis via multiple mechanisms.

Autophagy is a process involving the degradation and recycling of intracellular components to provide cellular energy 
and maintain nutritional support for cell survival (Singh and Cuervo, 2011). Previous studies reported that $H$. pylori infection induces autophagosome formation, and that autophagic vesicles provide special niches for $H$. pylori multiplication in both gastric epithelial cells (Terebiznik et al., 2009) and macrophages (Wang et al., 2009). Although $H$. pylori was protected in autophagosomes during the early stages of infection, the bacteria were degraded within the resulting autolysosomes of both cell types after $24 \mathrm{~h}$ of infection (Chu et al., 2010; Wang et al., 2010). Consistent with these findings, peripheral blood monocytes (PBMCs) harboring a single nucleotide polymorphism within the coding sequence for Autophagy Related 16-Like 1 (ATG16L1; ATG16L1300A), which is essential for autophagosome formation, exhibited impaired autophagy and increased susceptibility to $H$. pylori infection (Raju et al., 2012). In contrast, PBMCs harboring the ATG16L1300T allele showed a superior autophagic response and exhibited increased $H$. pylori clearance compared with PBMCs carrying the $300 \mathrm{~A}$ allele. These data imply that induction of autophagy might comprise a general mechanism for restricting bacterial replication in phagocytes (Deen et al., 2013).
In addition to lowering cellular cholesterol, statins were found to play a protective role in several bacterial infectious diseases. For example, while previous studies have established that Listeria monocytogenes triggers autophagy, resulting in reduced bacterial growth (Birmingham et al., 2007; Py et al., 2007), in a listeriolysin O (LLO)-dependent manner (MeyerMorse et al., 2010), statin treatment was found to enhance the host defense against L. monocytogenes by targeting LLOmediated phagosomal escape (Parihar et al., 2013). Furthermore, it was recently reported that statin therapy protects against Mycobacterium tuberculosis infection by enhancing autophagy and promoting phagosome maturation (Parihar et al., 2014). Consistent with these previous findings, our results demonstrate that statin treatment promoted autophagosome maturation and fusion with lysosomes, resulting in reduced bacterial burdens within macrophages, and thereby mitigated pathogenic infection by $H$. pylori (Figure 6).

There are several limitations to this study. First, only RAW264.7 macrophages and PEMs were utilized in our experiments; we have yet to test our findings in

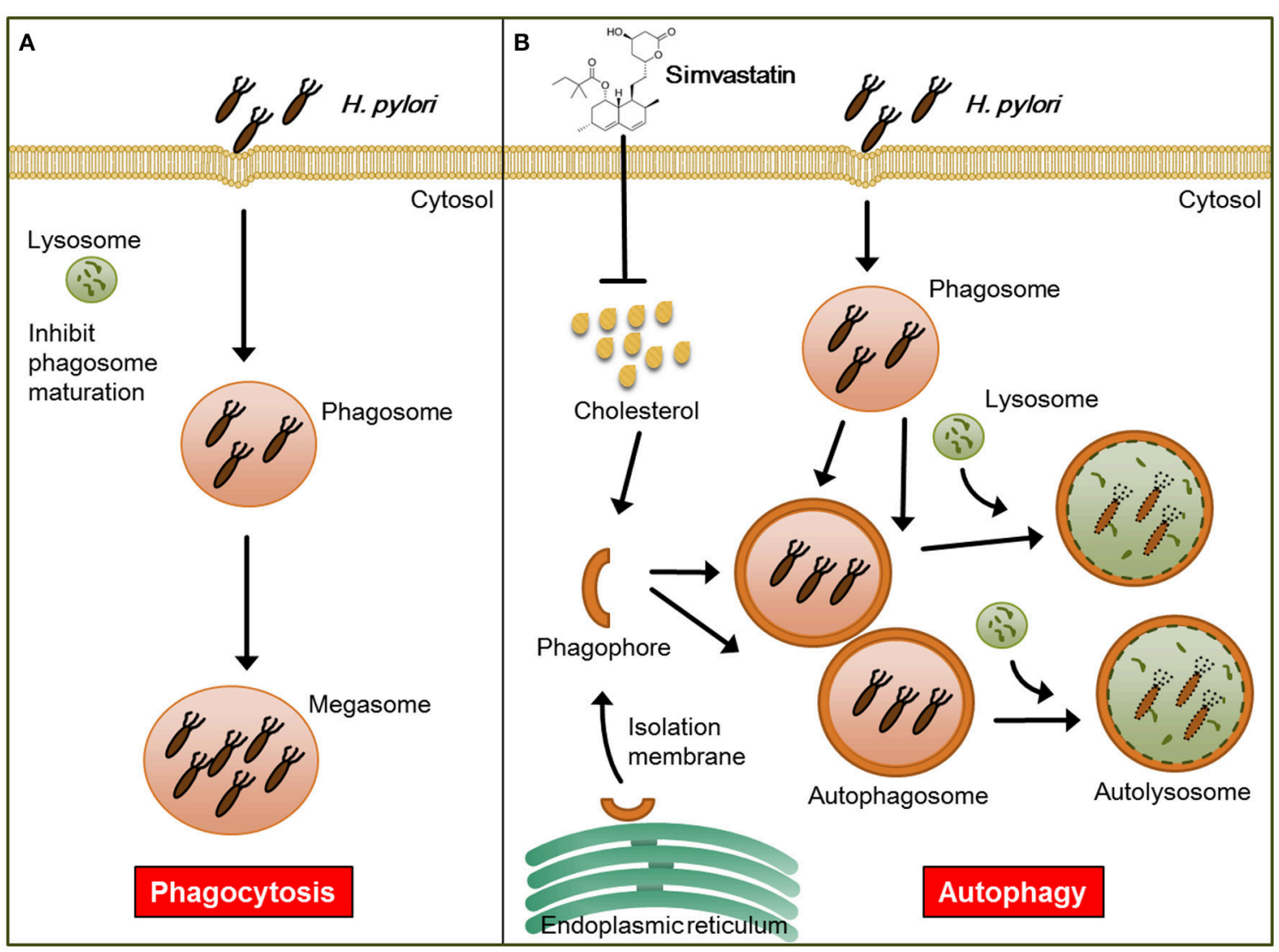

FIGURE 6 | Model for the mechanism by which statin enhances autophagy and alleviates pathogenic infection by Helicobacter pylori. (A) Infected macrophages harbor $H$. pylori, which inhibit phagosome maturation, within large vacuoles called megasomes. (B) Initiation of the autophagy pathway includes the translocation of the ULK complex from the cytosol to the endoplasmic reticulum (ER) and recruitment of the PI3K complex, which triggers the initiation of phagophore nucleation (Greenfield and Jones, 2013). This initiation process forms the isolation membranes, which in turn sequester cellular components and fuse to generate autophagosomes. Treatment of cells with simvastatin promotes the fusion of autophagosomes with lysosomes, leading to reduction in bacterial burdens and attenuation of $H$. pylori-induced inflammation. 
animal or human subjects. Likewise, the statin dosing regimens used in our cell-based study have yet to be evaluated in vivo. Therefore, further clinical investigations are required to clarify the link between statin use and regulation of autophagy. Such studies might pave the way for developing new strategies to control $H$. pylori infection.

In conclusion, the results of this investigation reveal that statin could potentially be used to decrease the intracellular burden of $H$. pylori in macrophages. In addition, statin enhanced early endosome maturation and subsequent activation of autophagy in macrophages, which promoted lysosomal fusion resulting in the degradation of sequestered $H$. pylori, followed by reductions in proinflammatory cytokine production. Future in vivo investigations and treatment regimens are needed to study the mechanism underlying the statin-mediated mitigation of $H$. pylori infection.

\section{AUTHOR CONTRIBUTIONS}

Conception or design of this work: WL, MH, and C-HL. Experimental study: WL, MH, MW, C-JL, TL, H-RL, and H-JL. Data analysis and interpretation: YS, MK, and YP. Writing the manuscript: WL, MH, and C-HL. Final approval: all authors

\section{FUNDING}

This work was supported by the Ministry of Science and Technology (104-2320-B-182-040 and 105-2313-B-182-001),

\section{REFERENCES}

Allen, L. A., Schlesinger, L. S., and Kang, B. (2000). Virulent strains of Helicobacter pylori demonstrate delayed phagocytosis and stimulate homotypic phagosome fusion in macrophages. J. Exp. Med. 191, 115-128. doi: 10.1084/jem.191. 1.115

Armitage, J. (2007). The safety of statins in clinical practice. Lancet 370, 1781-1790. doi: 10.1016/S0140-6736(07)60716-8

Birmingham, C. L., Canadien, V., Gouin, E., Troy, E. B., Yoshimori, T., Cossart, P., et al. (2007). Listeria monocytogenes evades killing by autophagy during colonization of host cells. Autophagy 3, 442-451. doi: 10.4161/auto. 4450

Boyd, A. R., Hinojosa, C. A., Rodriguez, P. J., and Orihuela, C. J. (2012). Impact of oral simvastatin therapy on acute lung injury in mice during pneumococcal pneumonia. BMC Microbiol. 12:73. doi: 10.1186/1471-2180$12-73$

Chan, L. L., Shen, D., Wilkinson, A. R., Patton, W., Lai, N., Chan, E., et al. (2012). A novel image-based cytometry method for autophagy detection in living cells. Autophagy 8, 1371-1382. doi: 10.4161/auto.21028

Cheng, J., Ohsaki, Y., Tauchi-Sato, K., Fujita, A., and Fujimoto, T. (2006). Cholesterol depletion induces autophagy. Biochem. Biophys. Res. Commun. 351, 246-252. doi: 10.1016/j.bbrc.2006.10.042

Chow, O. A., von Köckritz-Blickwede, M., Bright, A. T., Hensler, M. E., Zinkernagel, A. S., Cogen, A. L., et al. (2010). Statins enhance formation of phagocyte extracellular traps. Cell Host Microbe 8, 445-454. doi: 10.1016/j.chom.2010.10.005

Chu, Y. T., Wang, Y. H., Wu, J. J., and Lei, H. Y. (2010). Invasion and multiplication of Helicobacter pylori in gastric epithelial cells and implications for antibiotic resistance. Infect. Immun. 78, 4157-4165. doi: 10.1128/IAI.00524-10
Chang Gung Memorial Hospital (CMRPD1F0011-3, CMRPD1F0431-3, and BMRPE90), and the Tomorrow Medical Foundation.

\section{ACKNOWLEDGMENTS}

The authors would like to thank the editor and reviewers for the editorial assistance and their valuable comments. The authors sincerely appreciate the assistance of ShuChen Shen for analyzing confocal microscopy at the Division of Instrument Service of Academia Sinica, Taipei, Taiwan.

\section{SUPPLEMENTARY MATERIAL}

The Supplementary Material for this article can be found online at: http://journal.frontiersin.org/article/10.3389/fcimb. 2016.00203/full\#supplementary-material

Figure S1 | Statin does not affect the phagocytotic activity of macrophages. RAW264.7 cells treated with simvastatin $(0$ or $10 \mu \mathrm{M})$ for $8 \mathrm{~h}$ were incubated with latex beads, and phagocytosis activity was evaluated via flow cytometry analysis. The number at the right of each histogram represents the mean fluorescence intensity (MFI).

Figure S2 | Statin has no effect on extracellular bacterial adhesion to macrophages. RAW264.7 cells untreated or treated with $10 \mu \mathrm{M}$ simvastatin for 8 $\mathrm{h}$ were infected with Helicobacter pylori $(\mathrm{MOI}=100)$ for $6 \mathrm{~h}$, washed three times to remove unbound bacteria, and lysed by incubation in distilled water for $10 \mathrm{~min}$. Cell lysates were then plated onto Brucella blood agar plates and cultured for 3-5 days. The results are expressed as the mean ( \pm standard deviation) number of viable colony-forming units (CFU) obtained from three independent experiments.

de Chastellier, C., and Thilo, L. (2006). Cholesterol depletion in Mycobacterium avium-infected macrophages overcomes the block in phagosome maturation and leads to the reversible sequestration of viable mycobacteria in phagolysosome-derived autophagic vacuoles. Cell Microbiol. 8, 242-256. doi: 10.1111/j.1462-5822.2005.00617.x

Deen, N. S., Huang, S. J., Gong, L., Kwok, T., and Devenish, R. J. (2013). The impact of autophagic processes on the intracellular fate of Helicobacter pylori: more tricks from an enigmatic pathogen? Autophagy 9, 639-652. doi: 10.4161 /auto. 23782

Deretic, V., Saitoh, T., and Akira, S. (2013). Autophagy in infection, inflammation and immunity. Nat. Rev. Immunol. 13, 722-737. doi: 10.1038/nr i3532

Erkkilä, L., Jauhiainen, M., Laitinen, K., Haasio, K., Tiirola, T., Saikku, P., et al. (2005). Effect of simvastatin, an established lipid-lowering drug, on pulmonary Chlamydia pneumoniae infection in mice. Antimicrob. Agents Chemother. 49, 3959-3962. doi: 10.1128/AAC.49.9.3959-3962.2005

Fratti, R. A., Backer, J. M., Gruenberg, J., Corvera, S., and Deretic, V. (2001). Role of phosphatidylinositol 3-kinase and Rab5 effectors in phagosomal biogenesis and mycobacterial phagosome maturation arrest. J. Cell Biol. 154, 631-644. doi: $10.1083 /$ jcb.200106049

Greenfield, L. K., and Jones, N. L. (2013). Modulation of autophagy by Helicobacter pylori and its role in gastric carcinogenesis. Trends Microbiol. 21, 602-612. doi: 10.1016/j.tim.2013.09.004

Hutton, M. L., Kaparakis-Liaskos, M., Turner, L., Cardona, A., Kwok, T., and Ferrero, R. L. (2010). Helicobacter pylori exploits cholesterolrich microdomains for induction of NF-kappaB-dependent responses and peptidoglycan delivery in epithelial cells. Infect. Immun. 78, 4523-4531. doi: 10.1128/IAI.00439-10

Huynh, K. K., Eskelinen, E. L., Scott, C. C., Malevanets, A., Saftig, P., and Grinstein, S. (2007). LAMP proteins are required for fusion of 
lysosomes with phagosomes. EMBO J. 26, 313-324. doi: 10.1038/sj.emboj.760 1511

Jain, M. K., and Ridker, P. M. (2005). Anti-inflammatory effects of statins: clinical evidence and basic mechanisms. Nat. Rev. Drug Discov. 4, 977-987. doi: 10.1038/nrd1901

Kang, R., Zeh, H. J., Lotze, M. T., and Tang, D. (2011). The Beclin 1 network regulates autophagy and apoptosis. Cell Death Differ. 18, 571-580. doi: $10.1038 /$ cdd.2010.191

Klionsky, D. J., Abdalla, F. C., Abeliovich, H., Abraham, R. T., Acevedo-Arozena, A., Adeli, K., et al. (2012). Guidelines for the use and interpretation of assays for monitoring autophagy. Autophagy 8, 445-544. doi: 10.4161/auto. 19496

Lai, C. H., Chang, Y. C., Du, S. Y., Wang, H. J., Kuo, C. H., Fang, S. H., et al. (2008). Cholesterol depletion reduces Helicobacter pylori CagA translocation and CagA-induced responses in AGS cells. Infect. Immun. 76, 3293-3303. doi: 10.1128/IAI.00365-08

Lai, C. H., Hsu, Y. M., Wang, H. J., and Wang, W. C. (2013). Manipulation of host cholesterol by Helicobacter pylori for their beneficial ecological niche. BioMedicine 3, 27-33. doi: 10.1016/j.biomed.2012.12.002

Lai, C. H., Kuo, C. H., Chen, P. Y., Poon, S. K., Chang, C. S., and Wang, W. C. (2006). Association of antibiotic resistance and higher internalization activity in resistant Helicobacter pylori isolates. J. Antimicrob Chemother. 57, 466-471. doi: $10.1093 /$ jac/dki479

Lai, C. H., Poon, S. K., Chen, Y. C., Chang, C. S., and Wang, W. C. (2005). Lower prevalence of Helicobacter pylori infection with vacAsla, cagA-positive, and babA2-positive genotype in erosive reflux esophagitis disease. Helicobacter 10, 577-585. doi: 10.1111/j.1523-5378.2005.00363.x

Lai, C. H., Wang, H. J., Chang, Y. C., Hsieh, W. C., Lin, H. J., Tang, C. H., et al. (2011). Helicobacter pylori CagA-mediated IL-8 induction in gastric epithelial cells is cholesterol-dependent and requires the C-terminal tyrosine phosphorylation-containing domain. FEMS Microbiol. Lett. 323, 155-163. doi: 10.1111/j.1574-6968.2011.02372.x

Lai, C. K., Su, J. C., Lin, Y. H., Chang, C. S., Feng, C. L., Lin, H. J., et al. (2015). Involvement of cholesterol in Campylobacter jejuni cytolethal distending toxin-induced pathogenesis. Future Microbiol. 10, 489-501. doi: $10.2217 / \mathrm{fmb} .14 .119$

Levine, B., Mizushima, N., and Virgin, H. W. (2011). Autophagy in immunity and inflammation. Nature 469, 323-335. doi: 10.1038/nature09782

Lin, C. J., Lai, C. K., Kao, M. C., Wu, L. T., Lo, U. G., Lin, L. C., et al. (2015). Impact of cholesterol on disease progression. Biomedicine (Taipei). 5, 7. doi: 10.7603/s40681-015-0007-8

Lin, H. J., Hsu, F. Y., Chen, W. W., Lee, C. H., Lin, Y. J., Chen, Y. Y., et al. (2016). Helicobacter pylori Activates HMGB1 Expression and Recruits RAGE into lipid rafts to promote inflammation in Gastric Epithelial Cells. Front Immunol. 7:341. doi: 10.3389/fimmu.2016.00341

Lu, D. Y., Chen, H. C., Yang, M. S., Hsu, Y. M., Lin, H. J., Tang, C. H., et al. (2012b). Ceramide and Toll-like receptor 4 are mobilized into membrane rafts in response to Helicobacter pylori infection in gastric epithelial cells. Infect. Immun. 80, 1823-1833. doi: 10.1128/IAI.05856-11

Lu, D. Y., Tang, C. H., Chang, C. H., Maa, M. C., Fang, S. H., Hsu, Y. M., et al. (2012a). Helicobacter pylori attenuates lipopolysaccharide-induced nitric oxide production by murine macrophages. Innate Immun. 18, 406-417. doi: $10.1177 / 1753425911413164$

Mariño, G., Niso-Santano, M., Baehrecke, E. H., and Kroemer, G. (2014). Selfconsumption: the interplay of autophagy and apoptosis. Nat. Rev. Mol. Cell Biol. 15, 81-94. doi: 10.1038/nrm3735

Marshall, B. (2002). Helicobacter pylori: 20 years on. Clin. Med. 2, 147-152. doi: $10.7861 /$ clinmedicine.2-2-147

Meyer-Morse, N., Robbins, J. R., Rae, C. S., Mochegova, S. N., Swanson, M. S., Zhao, Z., et al. (2010). Listeriolysin $O$ is necessary and sufficient to induce autophagy during Listeria monocytogenes infection. PLoS ONE 5:e8610. doi: 10.1371/journal.pone.0008610

Mizushima, N., Levine, B., Cuervo, A. M., and Klionsky, D. J. (2008). Autophagy fights disease through cellular self-digestion. Nature 451, 1069-1075. doi: $10.1038 /$ nature 06639

Motzkus-Feagans, C. A., Pakyz, A., Polk, R., Gambassi, G., and Lapane, K. L. (2012). Statin use and the risk of Clostridium difficile in academic medical centres. Gut 61, 1538-1542. doi: 10.1136/gutjnl-2011-3 01378

Murata-Kamiya, N., Kikuchi, K., Hayashi, T., Higashi, H., and Hatakeyama, M. (2010). Helicobacter pylori exploits host membrane phosphatidylserine for delivery, localization, and pathophysiological action of the CagA oncoprotein. Cell Host Microbe 7, 399-411. doi: 10.1016/j.chom.2010.0 4.005

Nseir, W., Diab, H., Mahamid, M., Abu-Elheja, O., Samara, M., Abid, A., et al. (2012). Randomised clinical trial: simvastatin as adjuvant therapy improves significantly the Helicobacter pylori eradication ratea placebo-controlled study. Aliment Pharmacol. Ther. 36, 231-238. doi: 10.1111/j.1365-2036.2012.05161.x

Nseir, W., Khateeb, J., Tatour, I., Haiek, S., Samara, M., and Assy, N. (2010). Longterm statin therapy affects the severity of chronic gastritis. Helicobacter 15 , 510-515. doi: 10.1111/j.1523-5378.2010.00803.x

O'Connor, A., Molina-Infante, J., Gisbert, J. P., and O’Morain, C. (2013). Treatment of Helicobacter pylori infection 2013. Helicobacter 18(Suppl. 1), 58-65. doi: 10.1111/hel.12075

Parihar, S. P., Guler, R., Khutlang, R., Lang, D. M., Hurdayal, R., Mhlanga, M. M., et al. (2014). Statin therapy reduces the Mycobacterium tuberculosis burden in human macrophages and in mice by enhancing autophagy and phagosome maturation. J. Infect. Dis. 209, 754-763. doi: 10.1093/infdis/j it550

Parihar, S. P., Guler, R., Lang, D. M., Suzuki, H., Marais, A. D., and Brombacher, F. (2013). Simvastatin enhances protection against Listeria monocytogenes infection in mice by counteracting Listeria-induced phagosomal escape. PLoS ONE 8:e75490. doi: 10.1371/journal.pone.0075490

Py, B. F., Lipinski, M. M., and Yuan, J. (2007). Autophagy limits Listeria monocytogenes intracellular growth in the early phase of primary infection. Autophagy 3, 117-125. doi: 10.4161/auto.3618

Raju, D., Hussey, S., Ang, M., Terebiznik, M. R., Sibony, M., Galindo-Mata, E., et al. (2012). Vacuolating cytotoxin and variants in Atg16L1 that disrupt autophagy promote Helicobacter pylori infection in humans. Gastroenterology 142, 1160-1171. doi: 10.1053/j.gastro.2012.01.043

Schwartz, J. T., and Allen, L. A. (2006). Role of urease in megasome formation and Helicobacter pylori survival in macrophages. J. Leukoc. Biol. 79, 1214-1225. doi: 10.1189/jlb.0106030

Singh, R., and Cuervo, A. M. (2011). Autophagy in the cellular energetic balance. Cell Metab. 13, 495-504. doi: 10.1016/j.cmet.2011.04.004

Tariq, M., Khan, H. A., Elfaki, I., Arshaduddin, M., Al Moutaery, M., Al Rayes, H., et al. (2007). Gastric antisecretory and antiulcer effects of simvastatin in rats. J. Gastroenterol. Hepatol. 22, 2316-2323. doi: 10.1111/j.1440-1746.2007.05 021.x

Terebiznik, M. R., Raju, D., Vázquez, C. L., Torbricki, K., Kulkarni, R., Blanke, S. R., et al. (2009). Effect of Helicobacter pylori's vacuolating cytotoxin on the autophagy pathway in gastric epithelial cells. Autophagy 5, 370-379. doi: 10.4161/auto.5.3.7663

Wang, H. J., Cheng, W. C., Cheng, H. H., Lai, C. H., and Wang, W. C. (2012). Helicobacter pylori cholesteryl glucosides interfere with host membrane phase and affect type IV secretion system function during infection in AGS cells. Mol. Microbiol. 83, 67-84. doi: 10.1111/j.1365-2958.2011.0 7910.x

Wang, Y. H., Gorvel, J. P., Chu, Y. T., Wu, J. J., and Lei, H. Y. (2010). Helicobacter pylori impairs murine dendritic cell responses to infection. PLoS ONE 5:e10844. doi: 10.1371/journal.pone.0010844

Wang, Y. H., Wu, J. J., and Lei, H. Y. (2009). The autophagic induction in Helicobacter pylori-infected macrophage. Exp. Biol. Med. (Maywood). 234, 171-180. doi: 10.3181/0808-RM-252

Wroblewski, L. E., Peek, R. M. Jr., and Wilson, K. T. (2010). Helicobacter pylori and gastric cancer: factors that modulate disease risk. Clin. Microbiol. Rev. 23, 713-739. doi: 10.1128/CMR.00011-10

Wunder, C., Churin, Y., Winau, F., Warnecke, D., Vieth, M., Lindner, B., et al. (2006). Cholesterol glucosylation promotes immune evasion by Helicobacter pylori. Nat. Med. 12, 1030-1038. doi: 10.1038/n m1480

Yamato, M., Watanabe, T., Higuchi, K., Taira, K., Tanigawa, T., Shiba, M., et al. (2007). Anti-inflammatory effects of pravastatin on Helicobacter pylori-induced 
gastritis in mice. Dig. Dis. Sci. 52, 2833-2839. doi: 10.1007/s10620-006-9 638-7

Yang, J. C., and Chien, C. T. (2009). A new approach for the prevention and treatment of Helicobacter pylori infection via upregulation of autophagy and downregulation of apoptosis. Autophagy 5, 413-414. doi: 10.4161/auto.5. 3.7826

Zhao, Z., Fux, B., Goodwin, M., Dunay, I. R., Strong, D., Miller, B. C., et al. (2008). Autophagosome-independent essential function for the autophagy protein Atg5 in cellular immunity to intracellular pathogens. Cell Host Microbe. 4, 458-469. doi: 10.1016/j.chom.2008.1 0.003
Conflict of Interest Statement: The authors declare that the research was conducted in the absence of any commercial or financial relationships that could be construed as a potential conflict of interest.

Copyright (c) 2017 Liao, Huang, Wang, Lin, Lu, Lo, Pan, Sun, Kao, Lim and Lai. This is an open-access article distributed under the terms of the Creative Commons Attribution License (CC BY). The use, distribution or reproduction in other forums is permitted, provided the original author(s) or licensor are credited and that the original publication in this journal is cited, in accordance with accepted academic practice. No use, distribution or reproduction is permitted which does not comply with these terms. 